\title{
Fish Shape Recognition using Multiple Shape Descriptors
}

\author{
Moumita Ghosh \\ School of EducationTechnology \\ Jadavpur University \\ Kolkata,India
}

\author{
Joydeep Mukherjee \\ School of EducationTechnology \\ Jadavpur University \\ Kolkata,India
}

\author{
Ranjan Parekh,Ph.D \\ School of EducationTechnology \\ Jadavpur University \\ Kolkata,India
}

\begin{abstract}
This paper studies recognition of fish shapes using both Region based and Contour based shape based descriptors [9]. Moment Invariants are chosen as the Region based descriptor and the Simple (geometric) shape descriptors (SSD) are used as Contour based shape descriptors. The shapes are varied through scaling and rotation. Manhattan Distance is used as the classifier. The study of the recognition rate by using moment invariants and simple shape descriptors is done separately. Each moment invariant (M1, M2 , M3 , M4 and M5) is studied separately and jointly. Then simple shape descriptors are combined with moment invariants to get hybrid feature vectors for improving recognition rate.
\end{abstract}

\section{General Terms}

Fish Shape recognition

\section{Keywords}

Moment invariants, Eccentricity, Simple Shape Descriptors

\section{INTRODUCTION}

The number of digital images is growing rapidly, a consequence of the intense use of digital cameras, multimedia services etc. Imagery is being generated and maintained for a large variety of applications including art galleries, architectural and engineering design, geographic information systems, weather forecasting, medical diagnostics and law enforcement. Besides, in many areas, the use of image analysis has increased. In this situation, the ability to classify images is essential in order to manage and organize the collection of images on a database. Image search engines are generally based on textual metadata (e.g. file name, author, file size, creating date). So search results are dependent on textual captions and might not produce accurate results. Besides, manually labeling large databases of images is not feasible or very expensive.

In contrast, content-based image recognition (CBIR) [2] systems filter images based on their color, texture, shape or other features, providing better indexing and giving more accurate results. When an image is searched on the Internet, through search engines like Google and Yahoo, often, it is not possible to get the results of the expected image. As there may be an excessive amount of irrelevant records, finding an image on a database is an often complicated task. In contentbased image database systems, intrinsic properties of the query images are extracted in feature vectors which are indexed or compared to dataset images during query processing to find similar images from the database. Image classification is related to various areas.
This paper proposes a methodology to identify and recognize images in a CBIR scenario using shape descriptors. The organization of the paper is as follows: section 2 provides an overview of the previous works, section 3 outlines the proposed approach, section 4 details the experimentations done and results obtained, section 5 brings up the overall conclusions and future scopes.

\section{RELATED WORK}

In 1999, the authors, S. Abbasi, F. Mokhtarian, J. Kittler ,in their paper [2] have shown that the CSS (curvature scale space) representation and its matching algorithm can be used for the purpose of shape similarity retrieval in an affine transformed environment. In paper [4] , D. Zhang and G.Lu, have made a study and a comparison on four shape descriptors : Fourier descriptors (FD), curvature scale space (CSS) descriptors (CSSD), Zernike moment descriptors (ZMD) and grid descriptors (GD). It is concluded that the region based methods have poor performance than the contour based methods and retrieval performance of these shape descriptors depends on shape database. In [5] D. Zhang and G. Lu, propose a generic Fourier descriptor (GFD). The proposed shape descriptor is derived by applying 2-D Fourier transform on a polar raster sampled shape image and it is application independent and robust. Here, the authors conclude that the GFD performs better than Zernike moment descriptor (ZMD). In [6] D. Li \& S. Simske have proposed a shape retrieval algorithm. The algorithm is based on the geometry similarity theorem and Centroid-radii model. The distance ratio distribution is used to measure the similarity of the shapes. In paper [7], evaluation and comparison of the performance of Fourier descriptors and $\mathrm{Hu}$ 's seven moment invariants for recognizing images with different spatial resolutions is shown by the authors, Q. Chenl, E. Petriul and $X$. Yang. Fourier descriptors are boundary-based image features which only compute the pixels along the image contours. On the contrary, Hu's seven moment invariants are region-based. C. Shahabi and M. Safar, compare performance of boundary based methods for shape representation and retrieval: Fourier descriptors method FD (based on objects' shape radii), grid-based method GB (based on chain codes) in paper [10]. Combination of different percentages of five geometric shape descriptors are experimented by N. Jamil, et al. in paper [11] . This paper experiments on the combinations of the Eccentricity, Rectangularity, Compactness, Convexity, Solidity. In 2007, the authors, W. Lin, N. Boston ,Y. Hu [12], have experimented on the summation invariant of curves under transformation group action. In paper [13] a new approach for recognizing two-dimensional shapes called the SKS algorithm is investigated by K. Krish and W. Snyder in 2007. In the year, 2007, M. Sarfraz and A. Ridha, in their 
paper [14], present content-based image retrieval using various shape descriptors like 11 moment invariants, area ratios (3-concentric ring based and 8-sector based) and simple shape descriptors (eccentricity, compactness, convexity, rectangularity, and solidity). Euclidean distance and Cosine correlation coefficient are used for similarity measures. In the year 2007, the author S. A. Araujo , and H. Y. Kim [15], proposed a rotation, scaling, translation invariant shape recognition technique using morphological operations [16]. In [17], H. Y. Kim and S. A.S. Araújo, have proposed a technique,which is RST (rotation, scaling and translation) invariant shape recognition for noisy, grayscale images with inconstant background. In, 2011, J. Chaki and R. Parekh have proposed a recognition system for plant leaf images using moment invariants and centroid radii model[19]. In 2012, the authors S. Garg and G. S. Sekhon , in paper [20], have listed number of shape recognition techniques which have been defined previously and give a comparative analysis of various techniques. The author ,W. $\mathrm{Lu}$, in 2012, in his paper [21], has selected seven invariant moments, circularity degree, rectangle degree, sphericity degree, concavity degree and flat degree as description features. The author, D. Chaudhuri, in 2013, in his paper [22] ,have reviewed the existing shape representation and description techniques.The author has discussed several approaches of global contour and region based shape description methods.

\section{PROPOSED METHODOLOGY}

The paper proposes a system for recognizing 50 different types of fish shapes. Each class is represented by 10 images with transformed variations (rotated and scaled).There are some training and testing sets in each class. First, we calculate the class mean for training set of each class. Then for each samples, in each class of testing set, calculate the distance between the sample and the training set class mean .Now find the minimum distance among each sample of each class with each class mean and thus increase the count for each class having minimum distance .Then find the accuracy for each class and finally compute the combined accuracy.

The features are grouped into two categories: (1) moment invariants, and (2) Simple shape descriptors (SSD). For each group, the similarity measure is used i.e. Euclidean distance.

\subsection{Moment Invariants}

Invariant $\mathrm{I}$ is a function defined on the space of all admissible image functions that does not change its value under degradation operator $\mathrm{D}$, i.e. that satisfies the condition $\mathrm{I}(\mathrm{f})=$ $\mathrm{I}(\mathrm{D}(\mathrm{f}))$ for any image function $\mathrm{f}$. This property is called invariance [18] . M-K Hu [1] proposes 7 moment features that can be used to describe shapes and these are invariant to rotation, translation and scaling. For a digital image, the moment of a pixel $P(x, y)$ at location $(x, y)$ is defined as the product of the pixel value with its coordinate distances i.e. $m=x \cdot y \cdot P(x, y)$

The moment of the entire image is the summation of the moments of all its pixels. More generally the moment of order $(p, q)$ of image $I(x, y)$ is given by

$$
m_{p q}=\sum_{x} \sum_{y}\left\{x^{p} \cdot y^{q} \cdot I(x, y)\right\}
$$

$$
\begin{aligned}
& m_{00}=\sum_{x} \sum_{y}\left[x^{0} y^{0} I(x, y)\right]=\sum_{x} \sum_{y}[I(x, y)] \\
& m_{01}=\sum_{x} \sum_{y}\left[x^{0} y^{1} I(x, y)\right]=\sum_{x} \sum_{y}[y . I(x, y)] \\
& m_{10}=\sum_{x} \sum_{y}\left[x^{1} y^{0} I(x, y)\right]=\sum_{x} \sum_{y}[x . I(x, y)] \\
& m_{11}=\sum_{x} \sum_{y}\left[x^{1} y^{1} I(x, y)\right]=\sum_{x} \sum_{y}[x . y . I(x, y)] \\
& m_{20}=\sum_{x} \sum_{y}\left[x^{2} y^{0} I(x, y)\right]=\sum_{x} \sum_{y}\left[x^{2} . I(x, y)\right] \\
& m_{02}=\sum_{x} \sum_{y}\left[x^{0} y^{2} I(x, y)\right]=\sum_{x} \sum_{y}\left[y^{2} \cdot I(x, y)\right] \\
& m_{21}=\sum_{x} \sum_{y}\left[x^{2} y^{1} I(x, y)\right]=\sum_{x} \sum_{y}\left[x^{2} \cdot y \cdot I(x, y)\right] \\
& m_{12}=\sum_{x} \sum_{y}\left[x^{1} y^{2} I(x, y)\right]=\sum_{x} \sum_{y}\left[x \cdot y^{2} \cdot I(x, y)\right] \\
& m_{30}=\sum_{x} \sum_{y}\left[x^{3} y^{0} I(x, y)\right]=\sum_{x} \sum_{y}\left[x^{3} \cdot I(x, y)\right] \\
& m_{03}=\sum_{x} \sum_{y}\left[x^{0} y^{3} I(x, y)\right]=\sum_{x} \sum_{y}\left[y^{3} \cdot I(x, y)\right]
\end{aligned}
$$

The invariant moments which are invariant to rotation, are then defined as follows :

$$
\begin{aligned}
& \varphi_{1}=m_{20}+m_{02} \\
& \varphi_{2}=\left(m_{20}-m_{02}\right)^{2}+\left(2 m_{11}\right)^{2} \\
& \varphi_{3}=\left(m_{30}-3 m_{12}\right)^{2}+\left(3 m_{21}-m_{03}\right)^{2} \\
& \varphi_{4}=\left(m_{30}+m_{12}\right)^{2}+\left(m_{21}+m_{03}\right)^{2} \\
& \varphi_{5}=\left(m_{30}-3 m_{12}\right)\left(m_{30}+m_{12}\right)\left\{\left(m_{30}+m_{12}\right)^{2}\right. \\
&-3\left(m_{21}+m_{03}\right)^{2} \\
&+\left(3 m_{21}-m_{03}\right)\left(m_{21}\right. \\
&\left.+m_{03}\right)\left\{3\left(m_{30}+m_{12}\right)^{2}\right. \\
&\left.-\left(m_{21}+m_{03}\right)^{2}\right\} \\
& \varphi_{6}=\left(m_{20}-m_{02}\right)\left\{\left(m_{30}+m_{12}\right)^{2}-\left(m_{21}+m_{03}\right)^{2}\right\} \\
&+4 m_{11}\left(m_{30}+m_{12}\right)\left(m_{21}+m_{03}\right) \\
& \varphi_{7}=\left(3 m_{21}-m_{03}\right)\left(m_{30}+m_{12}\right)\left\{\left(m_{30}+m_{12}\right)^{2}\right. \\
&\left.-3\left(m_{21}+m_{03}\right)^{2}\right\} \\
&-\left(m_{30}-3 m_{12}\right)\left(m_{21}\right. \\
&\left.+m_{03}\right)\left\{3\left(m_{30}+m_{12}\right)^{2}\right. \\
&\left.-\left(m_{21}+m_{03}\right)^{2}\right\}
\end{aligned}
$$

To make the moments invariant to translation the image is shifted such that its centroid coincides with the origin of the coordinate system. The centroid of the image in terms of the moments is given by:

$$
x_{c}=\frac{m_{10}}{m_{00}}, \quad y_{c}=\frac{m_{01}}{m_{00}}
$$

Then the central moments are defined as follows:

$$
\mu_{p q}=\sum_{x} \sum_{y}\left\{\left(x-x_{c}\right)^{p} \cdot\left(y-y_{c}\right)^{q} \cdot I(x, y)\right\}
$$

To compute $\mathrm{Hu}$ moments using central moments the $\varphi$ terms in equation (3) need to be replaced by $\mu$ terms. It can be verified that

$$
\mu_{00}=m_{00}, \quad \mu_{10}=0=\mu_{01}
$$

To make the moments invariant to scaling the moments are normalized by dividing by a power of $\mu_{00}$. The normalized central moments are defined as below

$$
M_{p q}=\frac{\mu_{p q}}{\left(\mu_{00}\right)^{\omega}}, \text { where } \omega=1+\frac{p+q}{2}
$$

Based on the values of $p$ and $q$ the following are defined : 


\subsection{Simple Shape Descriptors (SSD)}

\subsubsection{Eccentricity $(e)$}

Eccentricity of an object can be defined as the eccentricity of the ellipse representing the unit-standard-deviation contour of its points [23]. Eccentricity of the boundary can also be defined as the ratio of the major to minor axis, calculated with moments using Equation 7. The value is always between 0 and 1.

$$
e=\frac{\left(\mu_{20}-\mu_{02}\right)^{2}+4 \mu_{11}{ }^{2}}{\left(\mu_{20}+\mu_{02}\right)^{2}}
$$

\subsubsection{Solidity $(s)$}

Solidity measures the density of an object. It is obtained as the ratio of the area of an image object and the area of the object's convex hull as in Eq. 8 [8]. Objects having irregular boundaries or containing holes have solidity less than 1 .

$$
s=\frac{\text { area }_{\text {image }}}{\text { area }_{\text {convexhull }}}
$$

\subsubsection{MajorAxisLength, MinorAxisLength}

Scalars specifying the length (in pixels) of the major axis and minor axis of the ellipse that has the same normalized second central moments as the region. This property is supported only for 2-D input label matrices.

\subsection{Classification}

Classification is done by dividing the dataset into a training set $T$ and a testing set $S$ each consisting of $n$ samples. The $i$-th training class $T_{i}$ is represented by the mean of the feature values of all its component samples.

$$
M_{i}=\frac{1}{n}\left\{T_{i 1}^{F}+T_{i 2}^{F}+\cdots+T_{i n}^{F}\right\}
$$

The $j$-th test sample $S_{j}$ with feature value $S_{j}^{F}$ is classified to class $k$ if the absolute difference $D_{j, i}$ between the $j$-th test sample and $i$-th training class is minimum for $i=k$ i.e.

$$
S_{j} \rightarrow k, \quad \text { if } D_{j, i}=\left|S_{j}^{F}-M_{i}\right| \text { is minimum for } i=k
$$

\section{EXPERIMENTATIONS \& RESULTS}

Experimentations are performed by using 500 fish shape images taken from the ShapeCN dataset [16]. The dataset is divided into 50 classes of 10 samples each, out of which 1 sample is used for training the system and the remaining 9 samples are used for testing. All images are saved in PNG format. The test samples are either a scaled or rotated version of the training sample. For each class, the first four test samples are derived by scaling the training sample by $20 \%$, $40 \%, 60 \%, 80 \%$ and the remaining 5 test samples are derived by rotating the training sample by 7 degrees, 35 degrees, 132 degrees, 201 degrees and 298 degrees. Fig. 1 depicts the training images of all 50 classes. Fig. 2 depicts the training (first image) and test images (remaining 9 images) for Class1.

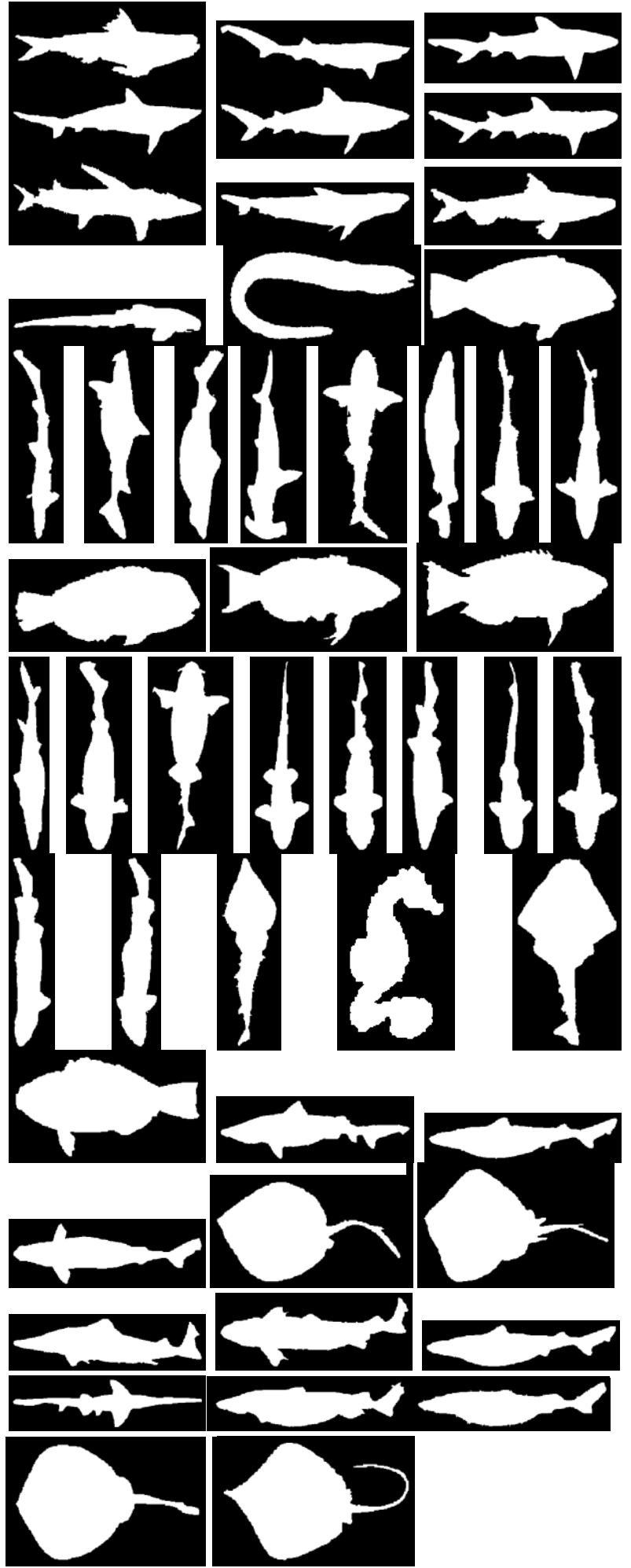

Fig 1: Training Images for all 50 classes 


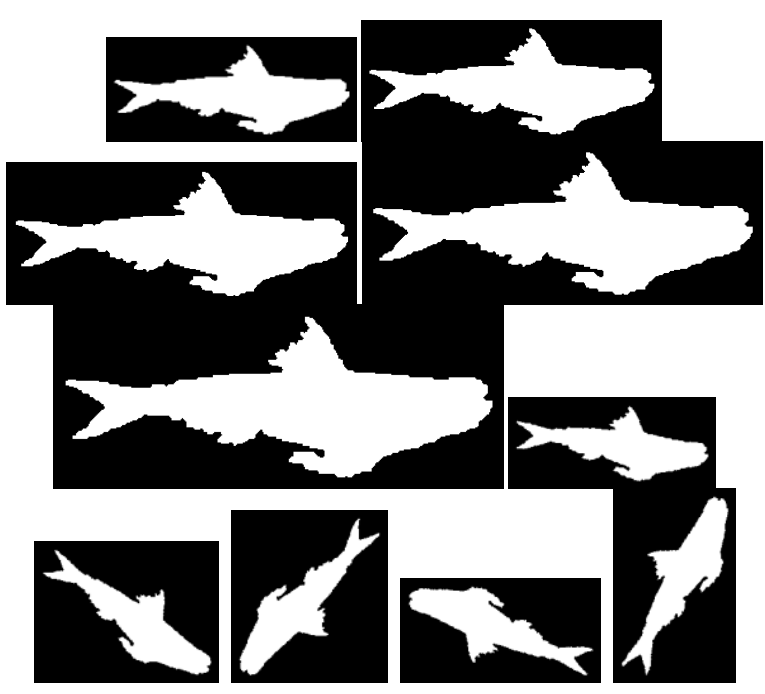

Fig 2: Training \& Testing Set Images for Class-1

\subsection{Moment-Invariants (M-I)}

\subsubsection{Individual Features}

Individual features values M1, M2, M3, M4 ,M5 for the training and testing images for the 50 classes are first used. Results are summarized in Table 1 below

Table 1. Recognition rate using individual feature

\begin{tabular}{|c|c|}
\hline Feature & Overall recognition rate (\%) \\
\hline M1 & 73.33 \\
\hline M2 & 15.55 \\
\hline M3 & 19.55 \\
\hline M4 & 19.55 \\
\hline M5 & 20.88 \\
\hline
\end{tabular}

\subsubsection{Joint Features}

To improve upon the results obtained using individual features, joint features are next considered in 2-D feature space. i.e. $\mathrm{M} 1+\mathrm{M} 2, \mathrm{M} 1+\mathrm{M} 3, \mathrm{M} 1+\mathrm{M} 4$. M1 is kept common. since it is seen to produce the best accuracies. Results are summarized in Table 2

Table 2. Recognition rate using joint features in 2D space

\begin{tabular}{|c|c|}
\hline Feature & Overall recognition rate (\%) \\
\hline M1+M2 & 70.44 \\
\hline M1+M3 & 73.33 \\
\hline M1+M4 & 73.33 \\
\hline M1+M5 & 73.33 \\
\hline
\end{tabular}

For the analysis of joint spaces, features are also considered in 3-D feature spaces : M1-M2-M3, M1-M2-M4, M1-M3-M4. Results are summarized on Table 3 below

Table 3. Recognition rate using joint features in 3D space

\begin{tabular}{|c|c|}
\hline Feature & Overall recognition rate (\%) \\
\hline $\mathrm{M} 1+\mathrm{M} 2+\mathrm{M} 3$ & 70.44 \\
\hline $\mathrm{M} 1+\mathrm{M} 2+\mathrm{M} 4$ & 70.44 \\
\hline $\mathrm{M} 1+\mathrm{M} 3+\mathrm{M} 5$ & 73.33 \\
\hline
\end{tabular}

\subsection{Simple Shape Descriptors (SSD)}

Table 4 indicates the accuracies obtained using four simple shape descriptors.

Table 4. Recognition rate using SSD

\begin{tabular}{|c|c|}
\hline Feature & Overall recognition rate (\%) \\
\hline Eccentricity & 79.11 \\
\hline MajorAxisLength & 28.88 \\
\hline MinorAxisLength & 28.44 \\
\hline Solidity & 45.55 \\
\hline
\end{tabular}

\subsection{Hybrid Features}

To study recognition accuracy of a hybrid set of features, combinations of simple shape descriptors and M-I features is used. Here, M1 gives the highest value among all moment invariants and also eccentricity gives highest recognition rate, so, the combination of $\mathrm{M} 1$ and eccentricity is done here, and obtains recognition rate of $90.88 \%$.

Table 5. Recognition rate using hybrid features

\begin{tabular}{|c|c|}
\hline Feature & Overall recognition rate (\%) \\
\hline M1 + Eccentricity & 90.88 \\
\hline
\end{tabular}

To provide a visual representation of the variation of these feature values over the classes, fig. 3 depicts variation of M1 and eccentricity (e) values of the training set images for all 50 classes. Fig. 4 depicts the corresponding plots for the testing set. In this case the mean value for all the 9 images per class have been used for plotting.
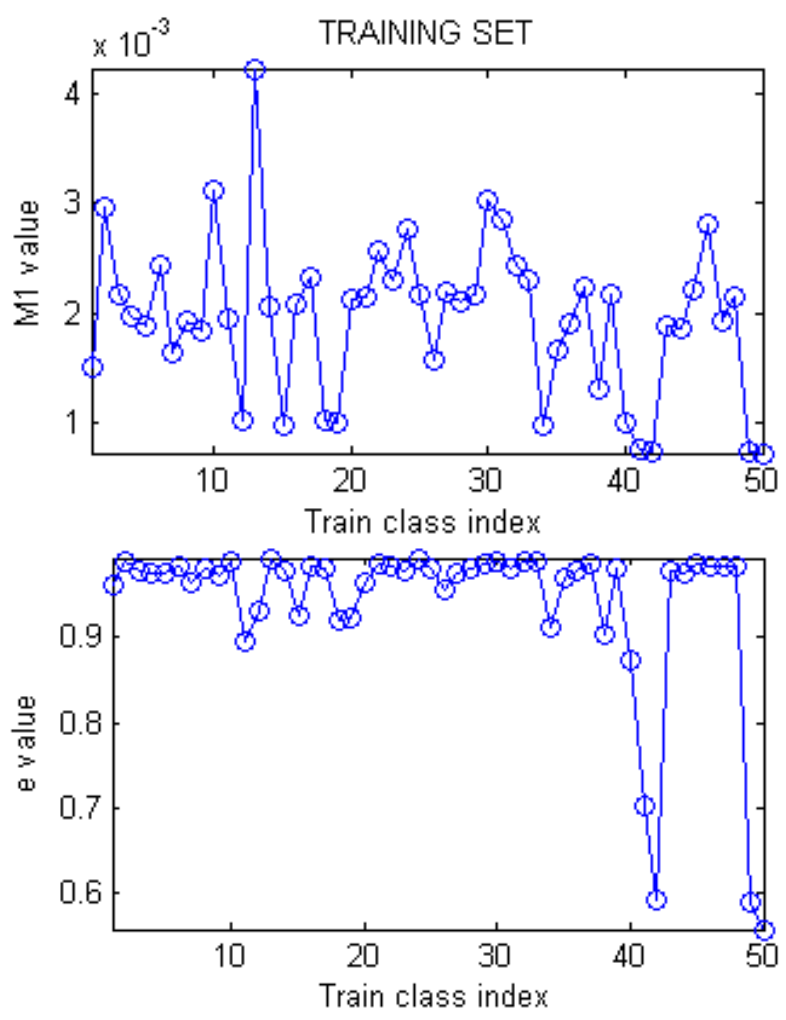

Fig 3: Feature plots for training set 

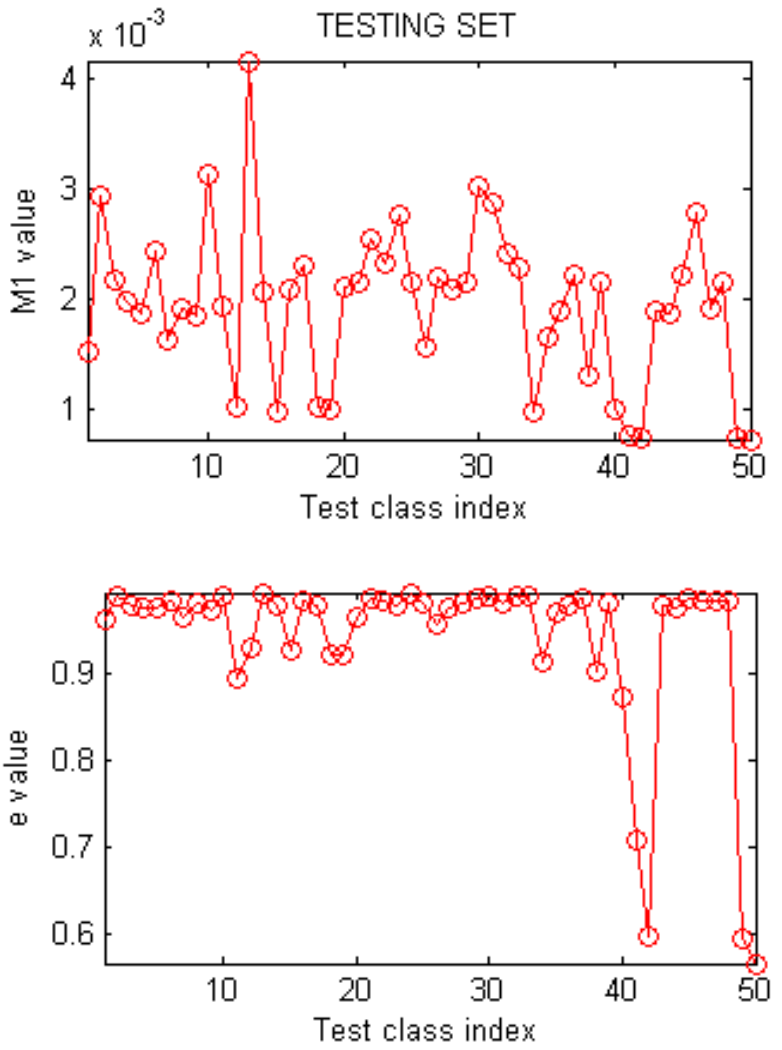

Fig 4: Feature plots for testing set

Fig. 5 depicts classification plots for four classes namely class-5, class-10, class-15, class-20. It shows that the difference between the mean values of the test samples of a specific class with the training samples of all 50 classes is minimum (indicated with a circle) when the training value corresponds to that specific class.
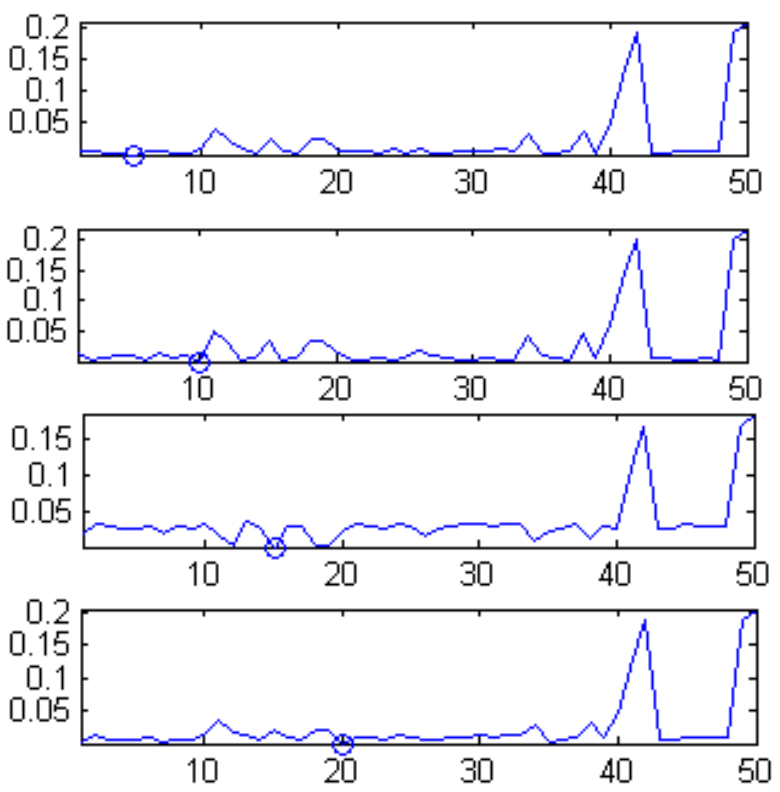

Fig 5: Classification plots for four classes
The percentage accuracies of all the 50 classes are shown in Fig. 6 . The accuracy values are : 100, 100, 100, 89, 89, 100 $100,100,67,100,100,100,100,100,100,100,100,89,56$, $100,100,78,100,100,22,100,100,89,100,100,100,100$, $100,100,100,100,89,100,67,100,100,78,100,100,11$, $100,100,100,44,78$

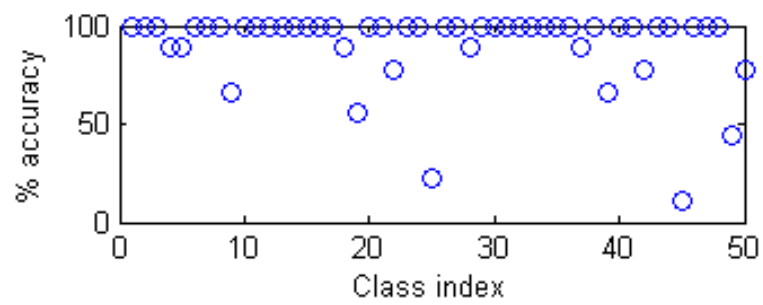

Fig 6: Percentage accuracies of all 50 classes

\section{CONCLUSIONS \& FUTURE SCOPES}

In this paper, transformation and scaling of the original image are taken into account. Experiments show that eccentricity gives the highest accuracy value. Also, M1 value surpass the other moment invariant values of $\mathrm{Hu}$. Combining M1 with eccentricity produces an improvement upon either of these features individually. The shape features and similarity measures used in this paper are experimented with binary images. Though, transformation and scaling are considered, but noise is not applied on the query image. Future work, can be involved by combining other shape based techniques like Hough transform and Fourier descriptors and can involve segmentation and other feature extraction methods.

\section{REFERENCES}

[1] $\mathrm{M}-\mathrm{K} \mathrm{Hu}$, "Visual pattern recognition by moment invariants", IRE Transactions on Information Theory, 1962, pp. 179-187.

[2] S. Abbasi, F. Mokhtarian, J. Kittler " Curvature scale space image in shape similarity retrieval",in Multimedia Systems 7: 467-476 (1999).

[3] Latecki, L.J. and Lakämper, R , 1999 Convexity rule for shape decomposition based on discrete contour evolution, Computer Vision and Image Understanding, ,73(3):441-454.

[4] D. Zhang and G.Lu, "Content-Based Shape Retrieval Using Different Shape Descriptors: A Comparative Study",in IEEE International Conference on Multimedia and Expo, 2001.

[5] D. Zhang and G. Lu," Generic Fourier Descriptor for Shape-based Image Retrieval", in International Conference on Multimedia \& Expo (Volume :1) IEEE, 2002.

[6] D. Li \& S. Simske, "Shape Retrieval Based on Distance Ratio Distribution", Technical Report, HP Laboratories, September 2002.

[7] Q. Chenl, E. Petriul, X. Yang'," A Comparative Study of Fourier Descriptors and Hu's Seven Moment Invariants for Image Recognition”, in IEEE,2004. 
[8] Zang D., Kamel, D.M. and Baciu , "Integrated Image \& Graphics Technologies", Kluwer International Series in Engineering and Computer Science, Boston Kluwer Academic Publishers, 2004.

[9] D.S. Zhang \& G. Lu, "Review of shape representation and description techniques", Pattern Recognition, vol. 37 no. $\mathrm{p}$;

1, pp. 1-19, January 2004.

[10] C. Shahabi , M. Safar, "An experimental study of alternative shape-based image retrieval techniques", in Springer Science + Business Media, LLC 2006

[11] N. Jamil, Z. Abu Bakar, \& T.M.T. Sembok, "Image Retrieval of Songket Motifs using Simple Shape Descriptors", in Proceedings of the Geometric Modeling and Imaging - New Trends, pp. 171-176, 2006.

[12] W. Lin, N. Boston ,Y. Hu ,'Summation invariant and its applications to shape recognition", 2007.

[13] K. Krish,W. Snyder ," A Shape Recognition Algorithm Robust to Occlusion: Analysis and Performance Comparison",IEEE, 2007.

[14] M. Sarfraz and A. Ridha, "Content-based Image Retrieval using Multiple Shape Descriptors”,IEEE, 2007.

[15] H. Y. Kim and S. A.S. Araújo "Rotation, scale and Translation invariant segmentation free shape recognition", Lecture Notes in Computer Science, vol. 4872 , pp. $100-113,2007$
[16] ShapeCN Dataset of the Scientific Computing Group,[fractal.ifsc.usp.br/dataset/ShapeCN.php]

[17] S. A. Araujo, and H. Y. Kim, "Rotation, scale and translation-invariant segmentation-free grayscale shape recognition using mathematical morphology," ISMM Int. Symp. Mathematical Morphology, 2007.

[18] Jan Flusser, Tomáš Suk and Barbara Zitová,"Moments and Moment Invariants in Pattern Recognition", 2009 John Wiley \& Sons, Ltd. ISBN: 978-0-470-69987-4.

[19] J. Chaki and R. Parekh,"Plant leaf recognition using shape based features and neural netwok classifiers",in IJACSA,vol. 2, No. 10,2011.

[20] S. Garg and G. S. Sekhon, "Shape Recognition Techniques: A Selected Review", in International Journal of Engineering Research \& Technology (IJERT) Vol. 1 Issue 4, ISSN: 2278-0181,2012

[21] W. Lu," Method for Image Shape Recognition with Neural Network", D. Jin and S. Lin (Eds.): Advances in CSIE, Vol. 2, AISC 169, pp. 547-55,2012.

[22] D. Chaudhuri, "Global Contour and Region Based Shape Analysis and Similarity Measures",in Defence Science Journal, Vol. 63, No. 1, January 2013, pp. 74-88.

[23] Wirth, M.A. Image processing algorithms and applications. Lecture Notes. Dept. of Computing and Informaton Science, University of Guelph, Ontario. url: 